\title{
Asociación entre el síndrome de Wolfram y la tetralogía de Fallot en una niña
}

\author{
Association of Wolfram syndrome with Fallot tetralogy in a girl
}

\author{
Dr. Hüseyin A. Korkmaz ${ }^{a}$, Dr. Korcan Demir ${ }^{a}$, Dra. Filiz Hazan ${ }^{b}$, Dra. Melek Yıldız ${ }^{a}$,Dra. Özlem N. Elmas ${ }^{a}$ y \\ Prof. Dr. Behzat Özkan ${ }^{a}$
}

\section{RESUMEN}

El síndrome de Wolfram(DIDMAOS: diabetes insípida, diabetes mellitus, atrofiaópticaysordera)esuntrastornoneurodegenerativo raro. Las mutaciones del gen WFS1 (wolframina) en el cromosoma 4 son responsables de las manifestaciones clínicas en la mayoría de los pacientes con síndrome de Wolfram. El síndrome de Wolfram también está acompañado por trastornos psiquiátricos, anomalías urodinámicas, movilidad articular limitada, neuropatía autónoma cardiovascular y gastrointestinal, hipogonadismo hipogonadotrópico en los varones y complicaciones microvasculares de la diabetes. Los datos acerca de las malformaciones cardíacas asociadas en los niños con síndrome de Wolfram disponibles en las publicaciones científicas son muy limitados. En este artículo presentamos el caso de una niña de 5 años con síndrome de Wolfram y tetralogía de Fallot.

Palabras clave: Síndrome de Wolfram, tetralogía de Fallot, DIDMAOS.

http:/ /dx.doi.org/10.5546/aap.2016.e163

\section{INTRODUCCIÓN}

Por primera vez en 1938, Wolfram describió este síndrome (DIDMAOS) en cuatro hermanos. Se trata de una afección clínica que ocasiona diabetes insípida, diabetes mellitus, atrofia óptica y sordera de manera concomitante. ${ }^{1}$ En general, inicialmente se diagnostica la diabetes mellitus, seguida de la atrofia óptica dentro de los primeros 10 años de vida, y diabetes insípida y sordera neurosensorial durante la segunda década.

a. Hospital de Formación e Investigación de Enfermedades y Cirugías Pediátricas Dr. Behçet Uz, Departamento de Pediatría, División de Endocrinología Pediátrica, Esmirna, Turquía.

b. Hospital de Formación e Investigación de Enfermedades y Cirugías Pediátricas Dr. Behçet Uz, Departamento de Genética, Esmirna, Turquía.

Correspondencia:

Dr. Hüseyin A. Korkmaz: drkorkmazanil@hotmail.com

Financiamiento: Ninguno.

Conflicto de intereses: Ninguno que declarar.

Recibido: 3-9-2015

Aceptado: 12-11-2015
Las mutaciones del gen WFS1 en el cromosoma 4 son responsables de las manifestaciones clínicas en la mayoría de los pacientes con síndrome de Wolfram. ${ }^{2}$ Este gen codifica una proteína de membrana del retículo endoplásmico (wolframina) presente en las neuronas, las células $\beta$-pancreáticas, el oído interno, el corazón, la placenta, los pulmones y el hígado. Si bien se desconoce la función de esta proteína, su deficiencia produce mayor estrés dentro del retículo endoplásmico, lo que conlleva al deterioro del ciclo celular y de la homeostasis del calcio. La wolframina ejerce una función en el mantenimiento de la homeostasis del retículo endoplásmico en las células $\beta$-pancreáticas. ${ }^{3}$

Raramente se han notificado malformaciones cardíacas en el síndrome de Wolfram, ${ }^{4-7}$ y tal vez sean responsables de la morbimortalidad causada por este trastorno. En este artículo presentamos un caso de síndrome de Wolfram acompañado por tetralogía de Fallot y analizamos los resultados, junto con referencias a las publicaciones científicas.

\section{CASO}

Se trataba de una niña de 5,3 años de edad con polidipsia y polaquiuria que había bajado $3 \mathrm{~kg}$ en el último mes. A los 4 meses de vida se le había diagnosticado tetratología de Fallot, reparada quirúrgicamente. Además se le realizó una audiometría, en la que se observó hipoacusia neurosensorial bilateral cuando tenía 3 años. En los últimos 4 meses, estaba recibiendo oxibutinina en dosis de $0,2 \mathrm{mg} / \mathrm{kg} /$ día para el tratamiento de la vejiga neurogénica. Se trataba de la segunda hija de padres aparentemente sanos y no consanguíneos, nacida a término mediante parto vaginal normal tras un embarazo sin complicaciones. En los antecedentes familiares no se observó diabetes mellitus.

Examen físico: peso: 14,9 kg (percentilo 3-10), talla: $122 \mathrm{~cm}$ (percentilo 50-75), estrabismo, y cicatrices quirúrgicas en el tórax. Análisis de laboratorio: glucosa plasmática de $314 \mathrm{mg} /$ dl, ausencia de cetonas en orina, concentración 
de péptido C de $0,18 \mathrm{pmol} / \mathrm{ml}(0,15-1,10) \mathrm{y}$ $\mathrm{HbA} 1 \mathrm{c}$ de $11,2 \%$. Se le diagnosticó diabetes mellitus. El nivel de anticuerpos contra la insulina era de 4,5 U (0-8 U), de anticuerpos anti-GAD, $0,9 \mathrm{U} / \mathrm{ml}(<1 \mathrm{U} / \mathrm{ml})$, y no se observaron anticuerpos anti-células de los islotes. Recibió el alta con la indicación de cuatro dosis de insulina regular $(0,73 \mathrm{U} / \mathrm{kg} /$ día $)$.

A los 7 años y medio, a pesar del adecuado control glucémico (el más reciente era $\mathrm{HbA1c}$ de $6,92 \%$ ), presentó polidipsia, polaquiuria y enuresis nocturna. La ingesta de líquidos se estimó en $3500 \mathrm{cc} / \mathrm{m}^{2} /$ día, y la diuresis, en $9,4 \mathrm{cc} /$ $\mathrm{kg} / \mathrm{h}$. Las pruebas funcionales renales (BUN: 8,9 $\mathrm{mg} / \mathrm{dl}$, creatinina: $0,37 \mathrm{mg} / \mathrm{dl}$ ) y los electrolitos séricos ( $\mathrm{Na}: 139 \mathrm{mmol} / \mathrm{L}, \mathrm{C}: 5,1 \mathrm{mmol} / \mathrm{L}$, $\mathrm{Cl}: 105 \mathrm{mmol} / \mathrm{L}$ ) eran normales. Examen de osmolalidad de la sangre: $275 \mathrm{mOsm} / \mathrm{kg}$, osmolalidad de la orina: $168 \mathrm{mOsm} / \mathrm{kg}$, y concentración de vasopresina en suero: $<0,5 \mathrm{pmol} / \mathrm{L}$. Se consideró que se trataba de diabetes insípida central y se instauró un tratamiento con aerosol nasal de desmopresina (10 $\mu \mathrm{g}$ dos veces al día). El uso de aerosol nasal de desmopresina mejoró marcadamente la polidipsia, la polaquiuria y la enuresis nocturna. Se le realizó un examen de fondo de ojos y se observó atrofia óptica bilateral, pero sin signos de retinopatía diabética. En la resonancia magnética de la hipófisis se notó la ausencia de una mancha brillante en el lóbulo posterior de la hipófisis. En consecuencia, se diagnosticó síndrome de Wolfram.
Se extrajo ADN genómico de los leucocitos de la sangre periférica del probando y de los padres usando el mini kit de ADN QIAamp (Qiagen, 51304, Düsseldorf, Alemania), conforme a los procedimientos estándar. Se amplificaron fragmentos genómicos, incluidas las regiones de codificación y las regiones intrónicas adyacentes del gen WFS1 mediante RCP, y empleando los partidores descritos anteriormente (fuente genética). Se purificaron y analizaron los amplicones mediante secuenciación cíclica con el kit secuenciador ABI BigDye Terminator v3.1 (ABI Applied Biosystems, Foster City, CA) en un secuenciador de ADN automático (ABI 3130 Genetic Analyzer, Applied Biosystems). En el probando, se detectó una mutación conocida (Y508fsX541, c.1523_1524delAT) homocigota en el exón 8 del gen WFS1 (Figura 1). Dicha mutación estaba presente en forma heterocigota en ambos padres.

\section{DISCUSIÓN}

Diversas alteraciones genéticas son responsables del síndrome de Wolfram. ${ }^{8}$ En la mayoría de los pacientes, las mutaciones del gen WFS1 son homocigotas o heterocigotas compuestas y producen una alteración en la función de la wolframina. Sin embargo, recientemente, se halló que una mutación del gen WFS1 dominante hereditaria era subyacente en el síndrome de Wolfram en una familia finlandesa. ${ }^{9}$ Se identificaron mutaciones en el
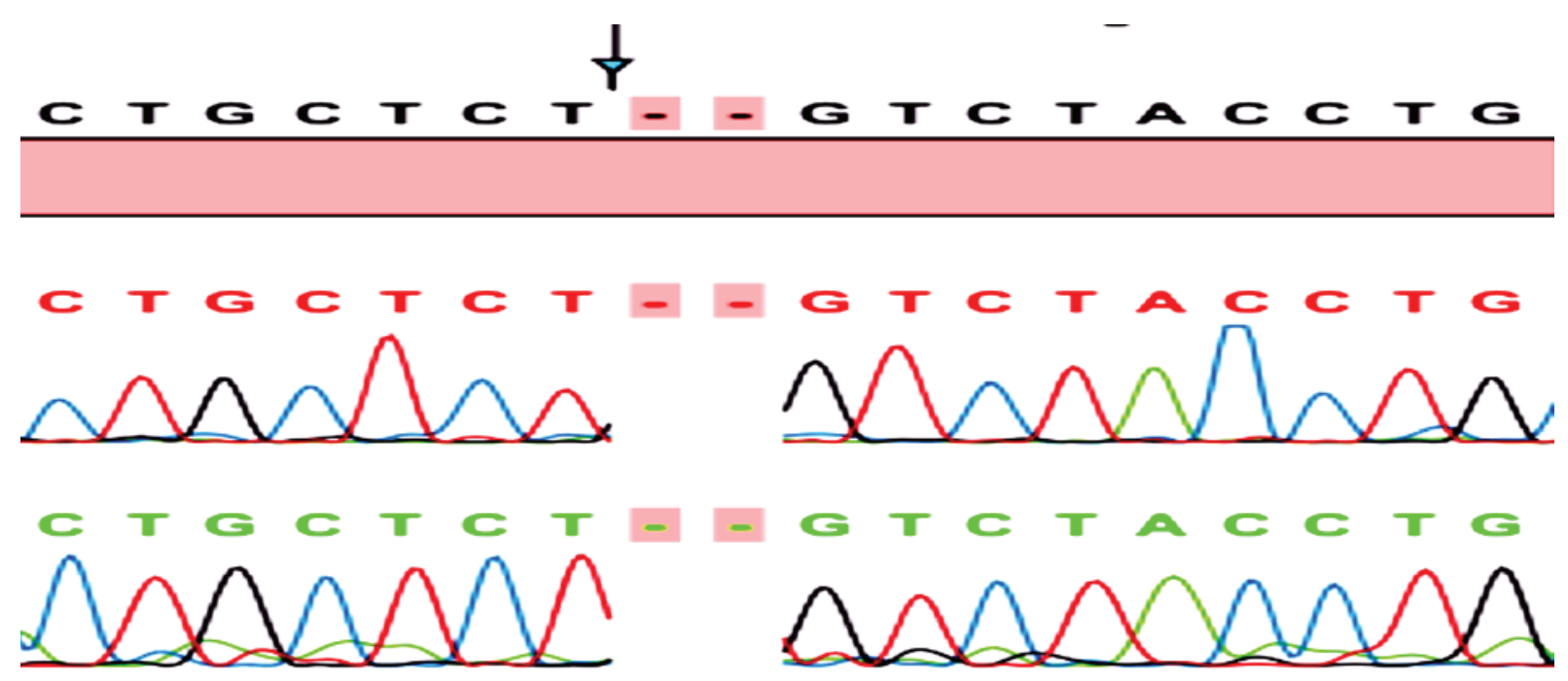
gen CISD2 en pacientes con un cuadro clínico similar, pero sin diabetes insípida (síndrome de Wolfram 2) ${ }^{10}$ De manera similar a la wolframina, la proteína intermembrana pequeña del retículo endoplásmico (endoplasmic reticulum intermembrane small protein, ERIS), codificada por el CISD2, se localiza en el retículo endoplásmico. ${ }^{10}$ En algunos pacientes con síndrome de Wolfram se demostraron mutaciones en el ADN mitocondrial. ${ }^{11,12}$ Los defectos mitocondriales que producen diabetes mellitus suelen estar acompañados por trastornos neurovasculares, que incluyen retraso mental, migraña, sordera y convulsiones. ${ }^{12}$ En el caso de nuestra paciente, el síndrome de Wolfram estaba asociado con una mutación homocigota en el gen WFS1.

La diabetes mellitus suele ser la primera manifestación clínica del síndrome de Wolfram y se presenta durante la primera década de vida, aproximadamente, y en promedio, a los 6 años de vida. Dado que no estaban presentes todas las características, no fue posible diagnosticar el síndrome de Wolfram en nuestra paciente al momento de aparición de la diabetes. Se sabe que el síndrome de rubéola congénita está asociado con la diabetes tipo 1, defectos cardíacos, sordera neurosensorial y cataratas. ${ }^{13}$ Nuestra paciente no tenía ninguno de estos antecedentes, cataratas ni autoanticuerpos asociados a la diabetes tipo 1 . Recientemente, se informó que las mutaciones en el gen GATA6 son la causa de cardiopatía congénita grave y de diabetes de inicio en el período neonatal o la infancia. ${ }^{14}$ Nuestra paciente no tenía insuficiencia pancreática exocrina ni anomalías hepatobiliares, que suelen producirse con las mutaciones en el gen GATA6. De manera retrospectiva, la presencia de cardiopatía congénita e hipoacusia neurosensorial en una niña sin autoanticuerpos con diabetes y disfunción miccional hubiera dado lugar al diagnóstico de síndrome de Wolfram al momento de la presentación, dado que, en estudios pertinentes, se ha notificado esta rara asociación.

En una serie de casos de 31 pacientes libaneses, se detectó valvulopatía, particularmente estenosis de la válvula pulmonar, en cinco pacientes $(16,1 \%)$ y comunicación interventricular en uno. ${ }^{4}$ En un informe de Turquía, se observó comunicación interventricular en algunos miembros de una familia afectada por el síndrome de Wolfram. ${ }^{5}$ En una serie de casos de 68 pacientes con síndrome de Wolfram, solamente tres tenían anomalías cardíacas: dos presentaban tetralogía de Fallot y uno, estenosis de la válvula pulmonar. ${ }^{6}$ No se habían realizado análisis mutacionales en ninguno de los pacientes antes mencionados con anomalías cardíacas. Aloi y col., informaron sobre un paciente con síndrome de Wolfram con tetralogía de Fallot sometida a reparación quirúrgica debido a una mutación heterocigota compuesta, c. $1628 \mathrm{~T}>\mathrm{G}$ y c. $2104 \mathrm{G}>\mathrm{A} .{ }^{7}$ En una serie de Strom y col., el paciente que tenía la mutación (c.1523delAT), como nuestra paciente, tenía un fenotipo grave con la aparición temprana de manifestaciones clínicas primordiales, entre otras, anomalías en el EEG y retraso de la madurez sexual. En el caso notificado, el hallazgo de una mutación en el exón 8 del gen WFS1 no estaba asociado con anomalía cardíaca. ${ }^{15}$ Nuestra paciente tenía vejiga neurogénica. En la serie de Colosimo y col., el paciente con la mutación c.1523delAT tenía un fenotipo leve y dilatación de las vías urinarias. ${ }^{11}$ Se desconoce el mecanismo subyacente de la asociación entre el síndrome de Wolfram y las malformaciones cardíacas. Estudios futuros podrían ayudarnos a comprender la función de la proteína wolframina en el desarrollo del sistema cardiovascular.

Una de las limitaciones de nuestro estudio fue no realizar la prueba de la sed para diagnosticar diabetes insípida según el examen de osmolalidad de la sangre y la orina. Se había planificado una prueba de la sed para establecer el diagnóstico de diabetes insípida, pero sus padres no aceptaron esta prueba. Se consideró que se trataba de diabetes insípida central, que tuvo una buena respuesta al tratamiento con aerosol nasal de desmopresina, ausencia de una mancha brillante en el lóbulo posterior de la hipófisis y mutación homocigota en el exón 8 del gen WFS1.

A modo de resumen, las cardiopatías congénitas pueden ser una de las características del síndrome de Wolfram. Esto es especialmente importante para el abordaje diagnóstico de los niños con diabetes sin autoanticuerpos y características incompletas del síndrome de Wolfram. El diagnóstico temprano y el manejo adecuado del paciente ayudarían a prevenir la aparición de complicaciones.

\section{REFERENCIAS}

1. Barrett TG, Bundey SE, Macleod AF. Neurodegeneration and diabetes: UK nationwide study of Wolfram (DIDMOAD) syndrome. Lancet 1995;346(8988):1458-63.

2. Hattersley AT, Njolstad P, Bruining J, Shield J, et al. Monogenic diabetes. En: International Diabetes Federation. Global IDF/ISPAD Guideline for Diabetes in Childhood and 
Adolescence. Brussels: International Diabetes Federation; 2011:31-6. [Acceso: 13 de noviembre de 2015]. Disponible en: http://www.idf.org/sites/default/files/Diabetes-inChildhood-and-Adolescence-Guidelines.pdf

3. Hilson JB, Merchant SN, Adams JC, Joseph JT. Wolfram syndrome: a clinicopathologic correlation. Acta Neuropathol 2009;118(3):415-28.

4. Medlej R, Wasson J, Baz P, Azar S, et al. Diabetes mellitus and optic atrophy: a study of Wolfram syndrome in the Lebanese population. J Clin Endocrinol Metab 2004;89(4): 1656-61.

5. Bekir NA, Gungör K, Güran S. A DIDMOAD syndrome family with juvenile glaucoma and myopia findings. Acta Ophthalmol Scand 2000;78(4):480-2.

6. Kinsley BT, Swift M, Dumont RH, Swift RG. Morbidity and mortality in the Wolfram syndrome. Diabetes Care 1995;18(12):1566-70.

7. Aloi C, Salina A, Pasquali L, Lugani F, et al. Wolfram syndrome: new mutations, different phenotype. PloS One 2012;7(1):e29150.

8. Khanim F, Kirk J, Latif F, Barrett TG. WFS1/wolframin mutations, Wolfram syndrome, and associated diseases. Hum Mutat 2001;17(5):357-67.

9. Bonnycastle LL, Chines PS, Hara T, Huyghe JR, et al.
Autosomal dominant diabetes arising from a wolfram syndrome 1 mutation. Diabetes 2013;62(11):3943-50.

10. Rigoli L, Di Bella C. Wolfram syndrome 1 and Wolfram syndrome 2. Curr Opin Pediatr 2012;24(4):512-7.

11. Colosimo A, Guida V, Rigoli L, Di Bella C, et al. Molecular detection of novelWFS1 mutations in patients with Wolfram syndrome by a DHPLC-based assay. Hum Mutat 2003;21(6): 622-9.

12. Hofmann S, Bezold R, Jaksch M, Kaufhold P, et al. Analysis of the mitochondrial DNA from patients with Wolfram (DIDMOAD) syndrome. Mol Cell Biochem 1997;174(1-2): 209-13.

13. Takasu N, Ikema T, Komiya I, Mimura G. Forty-year observation of 280 Japanese patients with congenital rubella syndrome. Diabetes Care 2005;28(9):2331-2.

14. Gong M, Simaite D, Kühnen P, Heldmann M, et al. Two novel GATA6 mutations cause childhood-onset diabetes mellitus, pancreas malformation and congenital heart disease. Horm Res Paediatr 2013;79(4):250-6.

15. Strom TM, Hörtnagel K, Hofmann S, Gekeler F, et al. Diabetes insipidus, diabetes mellitus, optic atrophy and deafness (DIDMOAD) caused by mutations in a novel gene (wolframin) coding for a predicted transmembrane protein. Hum Mol Genet 1998;7(13):2021-8. 